\title{
LA PRODUCTION DE VIANDE PAR L'EMBOUCHE DANS LES CONDITIONS TRADITIONNELLES AU NORD-CAMEROUN
}

\author{
E. ENGUELEGUELE
}

\begin{abstract}
RESUME
Au Nord Cameroun se pratique, dans la région de Margui-Wandala une embouche paysanne intensive en claustration dans des «cases".

On s'adresse à des taurillons de 2 à 3 ans. La nourriture d'abord d'herbe fine pendant les pluies est consutuée de sons de sorgho et de mil par la suite. Les taurillons prennent $100 \mathrm{~kg}$ en 10 à 12 mois puis sont abattus pour la fête du Maray.

Les commerçants pratiquent we embouche longue à 1 'herbe sur 2 ou 3 ans qui donne des animaux plus lourds.
\end{abstract}

\section{SUMMARY}

Meat production throught fattening under traditional conditions in Northern Cameroon

In Northern Cameroon, in the Margui-Wandala region, small farmer-managed intensive fattening is practiced on amimals shut-up in " huts ».

Feed consists of fine grass during rains, and of sorghum and millet brans latter on. The bullocks gain $100 \mathrm{~kg}$ in 10 to 12 months and are then slaughtered for the Maray feast.

Traders pratice long-term fattening on grass, during 2 or 3 years, thus obtaining heavy animals.

\section{Introduction et position du problème}

Le terme embouche désigne à la fois l'élevage des bovins pour la viande et la prairie où ces bovins s'engraissent.

Ainsi, notre confrère M. VILLEMIN (1) définit l'emboucheur comme une personne qui, possédant des herbages engraisse des bovins qu'il achète à bas prix; cette définition fait ressortir la notion fondamentale en rapport avec la pratique de l'embouche: c'est une notion de profit: achal d'animaux à bas prix pour les revendre plus cher après un temps plus ou moins long d'engraissement.

La notion de temps mis pour l'engraissement n'interviendra d'ailleurs que plus tard: elle a donné son essor à ce mode d'élevage, bénéficiant ainsi de l'évolution des sciençes de la nutrition et de l'économie rurale.

De nos jours, s'il fallait tenter de définir l'embouche, on pourrait dire que c'est le mode d'élevage qui consiste à apporter aux animaux achetés à bas prix une alimentation équilibrée et importante, dans le but de les engraisser pour la boucherie en un laps de temps relativement court.

\section{Caractéristiques de l'embouche artisanale au Nord-Cameroun}

Ainsi définie sous ses différents aspects, il est possible de reconnaitre deux formes d'embouche au Nord-Cameroun :
- les boufs de case du Margui-Wandala ;

- l'embouche par les commerçants à bétail dans les zones d'élevage et autour des grandes cités de l'Adamaoua.

\subsection{Les boufs de case du Margui-Wandala}

2.1.1. Limites géographiques de l'opéralion et motivations

Il existe, sur les «Massifs » de la région de Mokolo, une tradition d'embouche d'un type particulier.

Initialement, cette forme d'embouche, pratiquée par les Mofou des massifs de Douvangar, Douroum et Wazan, consistait à engraisser dans des cases, des jeunes taurillons destinés à être sacrifiés en offrande aux ancêtres à l'occasion de la Fête du Taureau ou Maray.

2.1.2. Technique de l'engraissenzent du "bouf de case $*$

\section{2-1.2.1. Description de la case:}

Le jeune taurillon, qui vient d'être acheté au marché de la plaine aux éleveurs foulbé, est âgé d'un à irois ans et pèse de 150 à $200 \mathrm{~kg}$.

Il est ramené par les petits chemins escarpés jusqu'à son étable, là-haut dans là montagne, où il est mis à l'engrais.

L'étable est une case particulière totalement obscure. En effet, ce local étroit n'est pourvu que d'une porte par laquelle a lieu la distribution des rations. L'animal n'en sortira que le jour de la Fête du Maray, 
après une phase d'engraissement de un an à plusieurs années.

L'écoulement du purin est facilité par une pente aménagée sur le sol et un orifice de communication avec l'extérieur.

La mangeoire en terre sur laquelle est entreposé le menu quotidien, est située du côté de la porte en surélévation.

\subsubsection{Alimentation:}

Ce taurillon mis à l'engrais a besoin d'un mois aut moins avant d'être habitué à l'alimentation intensive.

Le menu est composé d'herbe verte, mélange de graminées spontanées à prédominance de Pennisetum comptant théoriquement pour $0,14 \mathrm{UF}$ et $148 \mathrm{~g}$ de MPD/kg de M.S.

Cette herbe jeune est coupée sur les talus, mise en botte et transportée au village: l'animal peut en consommer 2 à 3 bottes en une journzée, selon l'âge. Les sons de sorgho et de mil, principaux déchets de cuisine sont apportés quotidiennement dans une petite calebasse réalisant ainsi un apport théorique de $0,85 \mathrm{UF} / \mathbf{k g}$ de M.S. Cet apport est d'ailleurs très limité, les sons étant surtout utilisés pour la fabrication de la bière; les drèches obtenues complètent, tout au long de l'année, la ration.

Il serait intéressant de faire le bilan de ce type d'alimentation de la saison pluvieuse: cette ration est, de toute évidence, abondante sur le plan énergétique et riche en matière azotée.

Pendant la saison sèche, les aliments disponibles sont représentés par le foin, coupé avant la fin des pluies et entreposé après fanage, les fanes d'arachides, sources importantes de matières protéiques digestibles, les tiges de mil rouge, les pieds de mil rabougri et les tiges de haricot. On continue a distribuer les sons de mil et de sorgho et les résidus des cuves de brassage de bière en quantités plus importantes.

\subsubsection{Importance numérique des laureaux ainsi préparés}

Jeanne-Françoise VINCENT, ethnologue, chargée de recherches au C.N.R.S., citée par le Révérend Père Yves SCHALLEZ, s'est intéressée aux implications sociologiques, aux difficultés et aux exigences propres de ce sacrifice. Elle nous aide à mesurer ]'importance attachée au sacrifice et rend compte du nombre de taureaux ainsi préparés.

Dans chacun des trois massifs cités plus haut, la Fête ne peut être célébréc que si le chef de massif en a domnć l'ordre: il faut "crier la Fête après avoir fait égorger chez lui le premier taureau et c'est alors que chaque homme adulte de son massif égorge son propre taureau qu'il avait mis à l'engrais dans son étable.

Cette Fête n'a d'ailleurs pas lieu en un seul jour; l'autorité des chefs de quartier relayant celle du chef du massif, elle va de quartier en quartier, se déroulant sur un mois environ, jusqu'à ce que le Prince du Massif la reprenme en main, en la clôturant par un sacrifice particulier, ce qui permet au Massif Mofou le plus proche et au chef qui le commande de prendre la relève.

Le nombre de taureaux ainsi préparés et égorgés peut donc être très important: Jeanne-Françoise VINCENT tapporte qu'en 1968, lors du Maray de Douvangar, plus de 100 boufs furent sacrifiés sur ce setrl massif.
Alain GAROUSTE quant à lui rapporte (6) qu'au cours du Maray de 1972, on a tué dans la région de Mokolo, 900 boeufs de case de 3 à 4 ans.

\subsubsection{Commentaires sur l'alimentation: performances} d'engraissement et importance économique actuelle

\subsubsection{Commentaires sur l'alimentation:}

Il șerait très intéressant de déterminer avec précision les quantités d'aliments consommés et le niveau de couverture des besoins de ces boufs de case au cours des deux saisons.

Ceci n'est malheureusement pas possible aujourd'hui, faute de données résultant d'études précises.

Il he fait cependant aucun doute que cette alimentation contient une quantité élevée d'énergie qui laisse pour l'engraissement un disponible non négligeable qui doit procurer un gain de poids correct.

\subsubsection{Performances d'engraissement et importance économique actuelle:}

Là encore, les données précises font défaut; on s'en tiendra aux estimations. Les taurillons de 3 ans sont mis à l'engrais pour l'année, c'est-à-dire pendant la saison de pluies et la saison sèche, période qui s'étend du début des pluies à la dernière campagne de récoltes qui intervient en octobre-novembre selon les années, soit une durée de 210 jours.

Pendant cette période, le poids des animaux passe de 250 à $350 \mathrm{~kg}$, soit un gain de $100 \mathrm{~kg} / 210$ jours. Ce qui donne, en grosse approximation, un gain de poids journalier de l'ordre de $500 \mathrm{~g}$, ce qui est correct.

Les commerçants à bétail représentent la clientèle de cette viande grasse très recherchée dans la région: la proportion de ces boufs dans les abattages globaux de la région serait de 10 p. 100, d'après les statistiques du Secteur Provincial d'Elevage et des Industries Animales du Nord.

Achetés 10 à $15000 \mathrm{~F}$, les animaux sont revendus 25000 à $30000 \mathrm{~F}$ après un ou deux ans d'engraissement.

\subsection{L'embouche des commerçants à bétail et des} intermédiaires

\subsubsection{Constitution du lot d'embotiche}

C'est le mode d'embouche le plus répandu à l'heure actuelle au Nord-Cameroun: il est surtout pratiqué par les marchands à bétail et les bouchers de l'Adamaoua.

Les taurillons sont achetés aux éleveurs traditionmels vers l'âge de 2 à 3 ans.

La plupart de ces commerçants sont également éleveurs au sens large du mot et castrent systématiquement les mâles à $2-3$ ans en vue de leur engraissement. Ces jeunes bouvillons seront dès lors retirés du ou des troupeaux et viendront s'ajouter aux taurilions d'achat du groupe d'âge identique, pour constituer le lot d'embouche.

\subsubsection{Alinnentation}

Tous ces animaux, castrés, déparasités, sont placés dans des pâturages riches avec d'importants basfonds à base d'hyparrhénia SP. Les charges sont parfois très élevées ( $500 \mathrm{~kg} /$ hectare); des blocs de natron disposés dans des pirogues creusées sur troncs d'arbres et du sel en nature apporté de façon périodique constituent le complément minéral. 
Le menu de saison des pluies est représenté par l'herbe de savane dont les repousses d'un mois autorisent un gain de poids.

En saison sèche, le facteur limitant est la faible valeur alimentaire des aliments récoltés, malgré le remplissage de la panse.

La possibilité d'exploitation d'un matériel riche sur brûlis demeure toutefois; elle ne dure qu'un moment et le pâturage devient vite râpé; en fin de compte, il faudra compter avec un préjudice important occasionné par la saison sèche: ces pertes expliquent la longueur du cycle de production. Nous y reviendrons.

\subsubsection{Durée de l'engraissement}

Les boufs ainsi engraissés sont livrés en boucherie à l'âge de 5 à 6 ans: l'opération a duré 2 à 3 ans. Ces boeufs, par leur qualité, sont destinés à l'exportation vers les villes du Sud et de l'Ouest; ce sont eux qui alimentent les courants d'exportation vers l'étranger.

Les carcasses de $300 \mathrm{~kg}$ ne sont pas exceptionnelles. Les travers de ce système d'embouche sont nombreux, retenons :

- la longueur du cycle de production: il faut attendre 5 à 6 ans pour produire un boeuf de 450 à $500 \mathrm{~kg}$;

- production abondante pendant l'hivernage (pcriode de vache grasse), déficit de production pendant la saison sèche et les deux mois qui suivent le retour des pluies.

\subsubsection{Importance économique de cette tradition et caractéristiques nouvelles de l'économie pastorale au Nord-Cameroun}

Il convient de signaler ici que, d'une façon générale, le contingent de boeufs commercialișćs au cours d'une année au départ de l'Adamaoua, par les professionnels de la viande au Cameroun, résulte d'un panachage entre les boufs provenant du système décrit plus haut et les boufs d'achat de 5 à 7 ans produits par les éleveurs dans les troupeaux cxtensifs.

Or, depuis quelques années, ces bceufs de 5 à 7 ans, engraissés dans les troupeaux extensifs, se font de plus en plus rares sur nos marchés locaux.

Danny DEEN et Dean JOHNSON, "Peace Corps" Américains qui ont étudié pendant deux ans la composition du troupeau de l'Adamaoua, estiment qu'ils ne représentent plus que 1,3 p. 100 du troupeau soit 15000 têtes.

Par contre, les mâles (bouvillons et taurillons) de 3 à 4 ans apparaissent de plus en píus sur les marchés. Or la réglementation vétérinaire en vigueur en interdit l'abattage.

Le panachage pose donc de nos jour, aux commercants à bétail, un problème de plus en plus difficile à résoudre et les amène à compter davantage sur les produits de leur système d'embouche artisanale.

Les statistiques du Secteur Centre estiment qu'en 1972 les 75 p. 100 de viande exportés (carcasses, bcufs sur pied) ont été ainsi produits.

Cette tendance va s'affermir avec le temps, ce qui sera la résultante d'un équilibre qui interviendra à long ou à moyen terme entre le cheptel du NordCameroun et le milieu. La production des animaux de boucherie au Cameroun sera de plus en plus basée sur le processus des transferts d'animaux.

En effet, l'éleveur camerounais tient à son système d'élevage traditionnel : l'élevage extensif restera donc pour longtemps encore la scule activité envisageable pour la majorité de nos éleveurs; il sera cependant amélioré ici et là par des actions prćcises sur le cheptel et l'cnvironzement et pourra évoluer en certains endroits vers le ranching.

Renforcement de la protection sanitaire, hydraulique pastorale, amélioration de la gestion des pâturages, éducation de l'éleveur, auront sur le cheptel des conséquences en chaînc, l'abottissement étant l'accroissement de celui-ci. Dès Iors, un équilibre sera atteint dans le milieu, équilibre où interviennent le taux de production des vaches qui sera augmenté, le pourcentage de mortalité des jetines qui sera diminué. Le pâturage restant inextensible, le projrriétaire qui ne peut plus garder dessus tout le troupeau, se débarrassera de préférence des jeunes animaux pour ne conserver que les adultes: l'âge de commercialisation sera ainsi progressivement réduit.

S'il n'est pas encore possible d'avancer de façon certaine que cet équilibre est atteint, force nous est cependant de constater aujourd'hui qu'en certains endroits, notre cheptel en accuse déjà les secousses annonciatriçes d'une situation nouvelle qui s'est amorcée. Ainsi, au Nord de la Bćnoué, par exemple, les parcours permettent de moins en moins de conserver le même eflectif: la vente des jeunes mâles de 2 à 4 ans devient, de l'avis de certains spécialistes de l'économic pastorale, une des caractéristiques de l'élevage du Nord-Cameroun. L'aflaire mérite qu'on s'y intéresse.

L'agriculteur, habitué à nourrix des boufs de labour à la tête d'une exploitation moderne avec herbage, cultures fourragères irriguées ou non, le commerçant à bétail ou le boucher autour des grands centres de la région, sont les relais indiqués du pasteur daisseur en assurant chez eux la finition de ces jeunes aniMain.

\subsubsection{Facteurs favorables à l'amélioration de cette embouche artisanale.}

L'importance croissante prise par l'cmbouche des intermédiaires et des bouchers dans le marché de la viande au Cameroun milite pour l'amélioration de ce système de production.

Quels sont donc les facteurs favorables à cette amelioration? Ils sont au nombre de trois:

- il y a d'abord les textes administratifs qui réglementent l'âge d'abattage des animaux, ces textes ont contribué, en imposant aux bouchers de garder plus longtemps en élevage les jeunes mâles d'achat, à améliorer le poids des carcasses;

- la présence en grande quantité, sur le marché, d'animaux à l'âge le plus favorable à l'engraissement;

- l'existence de sous-produits agro-industriels dans la région pour l'alimentation de finition de ces jeunes sujets en vue de raccourcir le cycle de production de la viande (tourteau de coton, farines basses de riz, mélasse...).

\section{Conclusion}

Dans le contexte du Nord-Cameroun, il apparait particulièrcment intéressant de mettre au point et de vulgariser des systèmes de finition de jetunes mâles de plus en plus nombreux sur nos marchés, en utilisant les sous-produits agro-industriels locaux. Cette possibilité est fondée sur des expériences pratiques menées ces derniers temps simultanément à la Station de Wakwa ct sur le terrain, et ayant fait leurs preuves. 


\section{BIBLIOGRAPHIE}

1. VILLEMIN (M.), Dictionnaire cle termes zootechniques et vétérinaires.

2. LACROUTS (M.), Aspects de la production et de la commercialisation du cheptel africain et malgache, I.E.M. V.T., 1964, Ens. n' IV, 22.

3. LHOSTE (P.), et DUMAS (R.), Varjation du poids vif et du rendement des boedis zébus de l'Adamaoua au cours de la saison seche, I.E.M.V.T. Wakwa.

4. PIOT (J.), Rapport anmuel: Section de Recherches Agrostologiques, I.E.M.V.T. Wakwa, 1964-1965 (Feux de brousse).

5. LHOSTE (P.), MICHEL (R.-J.), DUMAS (R.), Finition prócoce de boeuls zébus, I.E.M.V.T. Wakwa, aoûl 1970.

6. GAROUSTE (A), L'embouche dans le Margui-Wandala Projet d'amćlioration, 1973.

7. L’engraissement des zébus dans la région de Tananarive: Selon la technique du "Bळuf de Fosse", par 4 séries, Rev. Elev. Méd. vét. Pays trop., 1969, 22, 4, 429539 .

8. I.E.M.V.T. Wakwa: Bilan annuel d'activités pour 19711972 .

9. LHOSTE (PI.), PIERSON (J.-N.), GINISTY (L.), Essai de finition de boufs zébus avec utilisation maximale de jonélasse, I.E.M.V.T., aột 1973 